Реальная практика лекарственной терапии среднетяжелых и тяжелых форм воспалительных заболеваний кишечника в России, Республике Беларусь и Республике Казахстан. Промежуточные результаты исследования INTENT

\author{
Князев О.В. ${ }^{1}$ Белоусова Е.А. ${ }^{2}$ - Абдулганиева Д.И. ${ }^{3}$ • Губонина И.В. ${ }^{4}$ Кайбуллаева Д.А. ${ }^{5}$ \\ Мараховский Ю.Х. ${ }^{6}$ • Чашкова E.Ю.' • Шапина М.В. ${ }^{8}$ • Щукина O.Б. ${ }^{9}$ ' Гегенава Б.Б. ${ }^{10}$ • \\ Олиферук H.C. ${ }^{10}$
}

Актуальность. Анализ данных реальной клинической практики лечения больных воспалительными заболеваниями кишечника (ВЗК) эффективный инструмент совершенствования медицинской помощи этой категории пациентов. Исследования подобного рода в России проводятся редко, что затрудняет критическую оценку существующего положения и оптимизацию сложившейся практики.

Цель - изучить реальную практику медикаментозного лечения пациентов со среднетяжелым и тяжелым течением ВЗК в Российской Федерации, Республике Казахстан и Республике Беларусь.

Материал и методы. Проведен анализ промежуточных результатов исследования INTENT (NCT03532932) - международного многоцентрового ретроспективного и проспективного неинтервенционного наблюдательного исследования, проводимого в Российской Федерации, Республике Беларусь и Республике Казахстан. В ретроспективный анализ включены данные 706 пациентов старше 18 лет с подтвержденным диагнозом среднетяжелого/тяжелого язвенного колита (ЯК) и болезни Крона (БК), установленным не менее чем за 2 года до включения в исследование, и имевших обострение заболевания на момент включения в исследование или в предыдущие 2 года. Сбор данных проводили в рамках рутинной лечебной практики, при которой пациенты на момент включения в исследование получали стандартную терапию, включающую препараты 5-аминосалициловой кислоты (5-АСК), глюкокортикостероиды (ГКС) и иммуносупрессоры (ИС), а также генно-инженерные биологические препараты (ГИБП).
Результаты. Из 706 больных ВЗК 465 страдали ЯК и 241 - БК. Соотношение мужчин и женщин в обеих группах было одинаковым. Средний возраст пациентов с ЯК был выше, чем пациентов с БК: 41,8 (95\% доверительный интервал (ДИ) 40,6-43,0) года против 35,6 (95\% ДИ 33,9$37,3)$ года $(p=0,0001)$, как и средний возраст начала заболевания - 34,5 (95\% ДИ 33,29-35,61) против 29,7 (95\% ДИ 28,03-31,3) года $(p=0,0001)$ и средняя длительность заболевания с момента диагностики до включения в исследование - 7,3 (95\% ДИ 6,77-7,9) года в группе ЯК и 5,9 (95\% дИ 5,3-6,59) года в группе БК $(p=0,0027)$. Доля пациентов с семейным анамнезом ВЗК была низкой: 3,2 и 0,8\% соответственно $(p=0,0672)$. Курящих пациентов с БК было более чем вдвое больше, чем с ЯК (11,2\% против $5 \%, p<0,001)$. В группе больных ЯК работали $58,1 \%$, в группе пациентов с БК - 47,0\% $(p<0,05)$. Инвалидность по основному заболеванию имели $36,6 \%$ больных ЯК и 56,0\% больных БК $(\mathrm{p}<0,005)$.

Рецидивирующий характер течения заболевания отмечен у $72,9 \%$ при ЯК и 60,6\% при БК, у остальных пациентов заболевание носило непрерывный характер. Протяженность ЯК соответствовала в 58,9\% случаев панколиту, в 33,1\% - левостороннему колиту и в $8 \%$ - проктиту. По локализации БК распределение пациентов было следующим: илеоколит - 54,8\%, терминальный илеит - 23,7\%, колит - 20,3\%, изолированное поражение верхних отделов желудочно-кишечного тракта - 1,2\%. Частота осложненных форм при ЯК составила 12,9\%, при БК- 57,4\% ( $p=0,0001)$. Частота внекишечных проявлений при ЯК и БК не различалась ( 23,4 и $28,2 \%$ соответственно, $p=0,1705)$.
В исследовании были представлены различные паттерны терапии ЯК и БК. При ЯК схемы на основе препаратов группы 5-АСК \pm ГКС получали $25,4 \%$ больных, при БК - 3,7\% ( $\leq \leq 0,0001)$. Следующими по частоте схемами были: 5-АСК с последующим назначением ИС \pm ГКС (17,9\% при ЯК, 22,8\% при БК, p=0,1331); стандартная терапия (любой препарат 5-АСК, ИС или ГКС за исключением ГИБП) с последующим прекращением лечения либо сокращением до монотерапии ГКС (14,8\% при ЯК, 5\% при БК, $\mathrm{p}=0,0001)$; 5-АСК с последующей комбинацией с ИС, затем назначение любого ингибитора фактора некроза опухоли-альфа (иФНО-а) как основного препарата, но с сохранением 5-АСК и/или ИС (22\% при БК против 13,5\% при ЯК, p=0,0052); начало лечения с иФНО-а в комбинации с любым препаратом стандартной терапии без последующих изменений $(24,1 \%$ при БК и 13,6\% при ЯК, $p=0,0007)$. Реже применяли следующие терапевтические схемы: начальная терапия 5-АСК с последующим назначением иФНО-а при сохранении 5-АСК (4,5\% при ЯК и 2,5\% при БК, $\mathrm{p}=0,2181)$; начало лечения с иФНО-а в сочетании с любым препаратом стандартной терапии, затем отмена ГИБП при сохранении стандартной терапии или ее отмене $(4,3 \%$ при ЯК и 7,5\% при БК, $p=0,0812$ ).

Общая частота применения ГИБП на разных этапах лечения при БК $(66,4 \%)$ была существенно выше, чем при ЯК (39,4\%). Ведолизумаб при БК назначали чаще, чем при ЯК (в 10,4 и 3,4\% соответственно, $p=0,0003)$. Анализ практики применения ГКС выявил ряд негативных тенденций: половина пациентов с ВЗК получали более 2 курсов ГКС в течение двухлетнего периода, в ряде случаев - от 5 до 8 курсов. Средняя 
длительность курсов ГКС в большинстве схем как для ЯК, так и для БК колебалась в интервале 91-209 дней, что значительно превышает рекомендуемую продолжительность 12 недель (83 дня).

Заключение. По ряду демографических характеристик и клиническим особенностям исследуемая когорта пациентов с ВЗК соответствует глобальным мировым тенденциям: соотношение мужчин и женщин, средний возраст больных, молодой возраст начала заболевания, статус курения, частота внекишечных проявлений и локализация поражений при БК. Следует отметить присутствие 5-АСК почти во всех схемах лечения БК, что не соответствует рекомендуемой тактике лечения. Противоречит рекомендациям частое и длительное назначение ГКС. Обращает на себя внимание достаточно редкое и позднее назначение ГИБП, особенно при ЯК. Выявленные ошибки мы связываем с недостаточной информированностью врачей о современных подходах к терапии ВЗК, равно как и с низкой приверженностью пациентов к лечению.

Ключевые слова: воспалительные заболевания кишечника, болезнь Крона, язвенный колит, эпидемиология, лекарственная терапия, реальная клиническая практика, 5-аминосалициловая кислота, глюкокортикостероиды, иммуносупрессоры, генно-инженерные биологические препараты, ведолизумаб
Для цитирования: Князев $\mathrm{OB}$, Белоусова $\mathrm{EA}$ Абдулганиева ДИ, Губонина ИВ, Кайбуллаева ДА, Мараховский ЮХ, Чашкова ЕЮ, Шапина МВ, Щукина ОБ, Гегенава ББ, Олиферук НС. Реальная практика лекарственной терапии среднетяжелых и тяжелых форм воспалительных заболеваний кишечника в России, Республике Беларусь и Республике Казахстан. Промежуточные результаты исследования INTENT. Альманах клинической медицины. 2021;49(7):443-454. doi: 10.18786/20720505-2021-49-061.

Поступила 29.09.2021; доработана 24.10.2021; принята К публикации 30.10.2021; опубликована онлайн 16.12.202
Князев Олег Владимирович - д-р мед. наук, заведующий отделением воспалительных заболеваний кишечника'; ORCID: https://orcid.org/0000-0001-72500977

$\triangle 111123$, г. Москва, шоссе Энтузиастов, 86, Российская Федерация. Тел.: +7 (909) 6911016. E-mail: oleg@bk.ru

Белоусова Елена Александровна - д-р мед. наук, профессор, руководитель отделения гастроэнтерологии, заведующая кафедрой гастроэнтерологии факультета усовершенствования врачей²; ORCID: https://orcid.org/0000-0003-4523-3337. E-mail: eabelous@yandex.ru

Абдулганиева Диана Ильдаровна - д-р мед. наук, профессор, первый проректор, заведующая кафедрой госпитальной терапииз; ORCID: https://orcid.org/00000001-7069-2725. E-mail: diana_s@mail.ru
Губонина Ирина Владимировна - канд. мед. наук, доцент, доцент 2-й кафедры (терапии усовершенствования врачей)4; ORCID: https://orcid. org/0000-0002-6302-7767. E-mail: giv70@bk.ru

Кайбуллаева Джамиля Ахтановна - канд. мед. наук, доцент кафедры гастроэнтерологии ; ORCID: https://orcid.org/0000-0002-0783-4441. E-mail: kaibullaev@mail.ru

Мараховский Юрий Харитонович - д-р мед. наук, профессор клинической медицины, заведующий кафедрой гастроэнтерологии и нутрициологии ; ORCID: https://orcid.org/0000-0001-7327-7762.

E-mail: marakhouski@yahoo.co.uk

Чашкова Елена Юрьевна - канд. мед. наук, врач-колопроктолог, вед. науч. сотр., заведующая лабораторией реконструктивной хирургии научного отдела клинической хирургии ; ORCID: https://orcid. org/0000-0002-7953-6523.

E-mail: elenachash1027@yandex.ru
Шапина Марина Владимировна - канд. мед. наук, руководитель отдела по изучению воспалительных и функциональных заболеваний кишечника'; ORCID: https://orcid.org/0000-0003-1172-6221.

E-mail: shapina.mv@yandex.ru

Щукина Оксана Борисовна - д-р мед. наук, руководитель Центра воспалительных заболеваний кишечника․ E-mail: burmao@gmail.com

Гегенава Бадри Борисович - канд. мед. наук, медицинский советник ${ }^{10}$; ORCID: https://orcid.org/00000002-6701-9780. E-mail: badri.gegenava@takeda.com

Олиферук Наталья Сергеевна - канд. мед. наук, руководитель терапевтического направления гастроэнтерологии ${ }^{10}$.

E-mail: natalya.oliferuk@takeda.com

1ГБУЗ г. Москвы «Московский клинический научно-практический центр имени А.С. Логинова ДЗМ»; 111123, г. Москва, шоссе Энтузиастов, 86-6, Российская Федерация

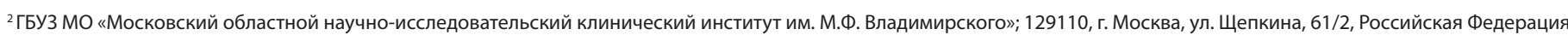
${ }^{3}$ ФГБОУ ВО «Казанский государственный медицинский университет» Минздрава России; 420012, г. Казань, ул. Бутлерова, 49, Российская Федерация ${ }^{4}$ ФГБВОУ ВО «Военно-медицинская академия имени С.М. Кирова» Минобороны России; 194044, г. Санкт-Петербург, ул. Академика Лебедева, 6А, Российская Федерация ${ }_{5}^{5}$ АО «Научно-исследовательский институт кардиологии и внутренних болезней»; 050000, г. Алматы, ул. Айтеке би, 120/25, Республика Казахстан

${ }^{6}$ ГУО «Белорусская медицинская академия последипломного образования»; 220013, г. Минск, ул. П. Бровки, 3-3, Республика Беларусь ${ }^{7}$ ФГБНУ «Иркутский научный центр хирургии и травматологии»; 664003, г. Иркутск, ул. Борцов Революции, 1, Российская Федерация

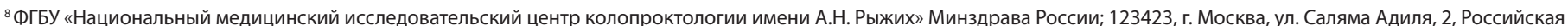
Федерация

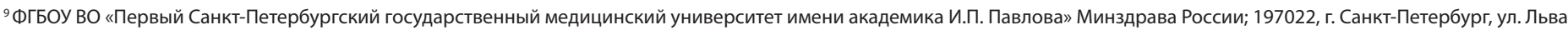
Толстого, 6-8, Российская Федерация

${ }^{10}$ ООО «Такеда Фармасьютикалс»; 119048, г. Москва, ул. Усачева, 2-1, Российская Федерация

$\mathrm{B}$ оспалительные заболевания кишечника (ВЗК) - группа неинфекционных полиэтиологичных хронических заболеваний желудочно-кишечного тракта (ЖКТ), которая включает язвенный колит (ЯК) и болезнь Крона (БК). ВЗК относятся к иммуноассоциированным заболеваниям. ЯК характеризуется воспалением слизистой оболочки толстой кишки, а БК - трансмуральным, сегментарным, гранулематозным воспалением различных отделов ЖКТ с развитием местных и системных осложнений. Течение ВЗК сопровождается периодами ремиссий и обострений. Для периода обострений типично развитие тяжелых симптомов со стороны 
ЖКТ и внекишечных проявлений (артропатии, аксиальные и периферические формы спондилоартрита, склерозирующий холангит, поражения слизистых оболочек и др.), которые могут значительно снизить качество жизни пациента или привести к инвалидности. Для достижения контроля над заболеванием необходимо пожизненное лечение. Учитывая, что эти заболевания в большинстве случаев поражают трудоспособную часть населения, актуальность данной проблемы высока $[1,2]$.

По имеющимся данным, распространенность ВЗК в России составляет от 19,3 до 29,8 на 100 тыс. населения для ЯК и от 3,0 до 4,5 на 100 тыс. населения для БК [3]. Однако распространенность ВЗК довольно сильно варьирует в зависимости от региона. Так, в Московской области этот показатель равен 58 на 100 тыс. населения, в Республике Татарстан - 40 на 100 тыс., в Новосибирской области - 49 на 100 тыс. [3], в Иркутской области 74,9 на 100 тыс. [3, 4]. В Республике Беларусь, по статистическим данным за 2016 г., распространенность ЯК составила 36,4 на 100 тыс., БК - 8,1 на 100 тыс. [5]. В Казахстане аналогичные показатели за 2019 г. были 6,3 и 31,5 на 100 тыс. соответственно [6].

Отсутствие единого регистра ВЗК затрудняет объективную оценку сложившейся практики терапии и совершенствование подходов к лечению. Целенаправленное и полноценное изучение реальной клинической практики лечения самой проблемной группы пациентов с ВЗК в Российской Федерации, Беларуси и Казахстане было предпринято в рамках исследования INTENT. Исследования подобного рода в России и странах СНГ проводятся редко, что затрудняет реализацию потенциала критической оценки и оптимизации сложившейся практики [7-9].

Целью исследования был сбор информации о ведении в реальной клинической практике пациентов со среднетяжелыми и тяжелыми ВЗК в Российской Федерации, Республике Беларусь и Республике Казахстан - документально зафиксировать схемы лечения и результаты лечения у пациентов с ВЗК, в том числе получающих биологические препараты.

\section{Материал и методы}

INTENT (NCT03532932) - международное многоцентровое ретроспективное и проспективное неинтервенционное наблюдательное клиническое исследование, спланировано и проводится в соответствии с принципами проведения клинических исследований, сформулированными в Декларации Всемирной медицинской ассоциации, принятой в Хельсинки в 1964 г. [10]. В исследовании принимали участие 36 медицинских центров, в том числе 31 центр из Российской Федерации, 2 из Беларуси и 3 из Казахстана. Протокол исследования одобрен локальными этическими комитетами каждого медицинского центра - участника исследования. Набор пациентов стартовал 20 июля 2018 г. и был прекращен в октябре 2020 г., окончание сбора данных планируется в январе 2022 г., и к июню 2022 г. ожидается подготовка финального отчета о проведении исследования с последующим анализом и публикацией результатов.

Исследование проведено при организационной и финансовой поддержке компании «Такеда».

Планируемое число участников - 2000. В данной работе приводятся промежуточные результаты анализа по 706 больным ВЗК.

Критерии включения:

- пациенты мужского и женского пола в возрасте 18 лет или старше на момент включения;

- подтвержденный диагноз БК или ЯК в течение по меньшей мере 2 лет до включения в исследование;

- наличие среднетяжелого или тяжелого обострения ВЗК на момент включения в исследование либо в анамнезе за предшествующие 2 года до включения в исследование, по поводу которого назначалась терапия кортикостероидами и/или иммуносупрессорами и/или биологическая терапия (обострение ВЗК должно быть документально подтверждено);

- проводимая в настоящее время терапия кортикостероидами, и/или иммуносупрессорами, и/или препаратами 5-аминосалициловой кислоты (5-АСК), и/или биологическая терапия.

Bсе участники подписывали информированное согласие на включение в настоящее исследование.

Дизайн исследования предполагал ретроспективный сбор данных о практике лечения пациентов в течение 2 лет до момента включения в исследование, а также проспективное наблюдение в течение 1 года со сбором данных на момент включения в исследование, через 6 и 12 месяцев после включения.

Первичными конечными точками данного промежуточного анализа было распределение схем лечения, используемых у пациентов со среднетяжелым и тяжелым ЯК и среднетяжелой и тяжелой БК, получающих биологические и стандартные препараты. Вторичные конечные точки 
включали распределение социодемографических, клинических показателей, описание методов определения активности заболевания и частоты объективной оценки активности ЯК/БК, анализ использования ресурсов здравоохранения.

В ходе исследования собирали сведения о демографических характеристиках участников, характере течения ВЗК, практике медикаментозного лечения, а также объемах стационарной медицинской помощи.

Ниже представлены промежуточные результаты, основанные на ретроспективном анализе части когорты участников в период 2 лет до включения в исследования, данные по которым были обработаны к 15 марта 2021 г. Ретроспективный дизайн означает, что данные всех пациентов, которые собраны в исследовании, были указаны в медицинской карте пациента до даты подписания формы информированного согласия (в рамках ответственности исследователя за конфиденциальность и защиту данных, описанных в протоколе исследования).

Информация по пациентам с БК и ЯК дана отдельно. Учитывали демографические характеристики пациентов, историю ВЗК, сопутствующие заболевания, историю применения биологических лекарственных средств, а также аминосалицилатов, кортикостероидов и иммунодепрессантов, локализацию патологического процесса и другие характеристики. Комбинации лекарственных препаратов, назначаемых для лечения, изучали отдельно для ЯК и БК. Каждую комбинацию лечения анализировали с использованием следующих показателей: средняя продолжительность курса, средняя максимальная дозировка каждого компонента, среднее количество курсов и др. Под паттерном терапии понимали уникальную комбинацию определенных вариантов лечения, а также их изменение с течением времени. В данном промежуточном анализе паттерны терапии выделяли ретроспективно, в период до 2 лет до включения в исследования, a также непосредственно на момент включения в исследование.

Для оценки данных и результатов исследования в разных подгруппах использовали описательный анализ. Описательная статистика включала сводные таблицы (для непрерывных переменных - $n$, среднее значение, медиана, стандартное отклонение, минимальное и максимальное значение, нижний и верхний квартиль; для категориальных значений - $n$, частота и процентная доля). Доля оценивали вместе с 95\% доверительным интервалом, если это применимо.
Для определения статистической значимости полученных результатов между подгруппами пациентов с ЯК и БК использовали анализ таблиц сопряженности частот $2 \times 2$ с применением точного теста Фишера, расчет Т-критерия Стьюдента с оценкой значимости $p$. За уровень статистической значимости показателей принято $\mathrm{p}<0,05$. Статистический анализ проводили с использованием программного пакета Microsoft R Open версии 3.x.х от Microsoft R Application Network (MRAN) (https://mran.microsoft.com).

\section{Результаты}

Исходная клинико-демографическая характеристика пациентов

В рамках промежуточного анализа были обработаны данные 706 пациентов с В3К: 465 (65,9\%) с ЯК, $241(34,1 \%)$ с БК. Как видно из данных табл. 1, средний возраст пациентов с БК был несколько меньше, чем у больных с ЯК (35,6 и 41,8 года соответственно), мужчин и женщин в обеих группах было практически поровну. Примерно половина пациентов имела постоянную трудовую занятость. Семейный анамнез ВЗК был отмечен лишь у небольшого числа больных, однако при ЯК он встречался втрое чаще, чем при БК (3,2 и 0,9\% coответственно).

Длительность заболевания до включения в исследование составила 7,34 года в группе ЯК и 5,94 года в группе БК. Средний возраст манифестации заболевания был 34,5 года в группе больных ЯК и 29,7 года - БК (см. табл. 1).

У всех больных наблюдалось хроническое течение заболевания в соответствии с критериями включения в исследование, при этом у большинства пациентов (72,9\% при ЯК и 60,6\% при БК) отмечен рецидивирующий характер течения, у остальных заболевание носило непрерывный характер (табл. 2).

Протяженность ЯК соответствовала в сумме панколиту/ левостороннему колиту в $92 \%$ и лишь в $8 \%$ - проктиту. Это обусловлено тем, что пациенты легкой степени тяжести, согласно дизайну, не включались в исследование. Что касается локализации воспалительного процесса при БК, более половины всех случаев представлены илеоколитом.

Частота осложнений в анамнезе в исследуемый период была более чем в 3 раза выше при БК по сравнению с ЯК. Частота внекишечных проявлений при ЯК и БК практически не различалась (см. табл. 2).

Доля курящих пациентов с БК была вдвое выше, чем с ЯК (11,2 и 5\% соответственно, рис. 1). 
Таблица 1. Демографические характеристики участников на момент включения в исследование INTENT

\begin{tabular}{|c|c|c|c|c|c|}
\hline Показатель & $\begin{array}{l}\text { Язвенный колит } \\
\quad(\mathrm{n}=465)\end{array}$ & 95\% ди & Болезнь Крона $(n=241)$ & 95\% ди & Значение $p$ \\
\hline Женщины, абс. (\%) & $215(46,2)$ & $41,6-50,9$ & $121(50,2)$ & $43,7-56,7$ & 0,3405 \\
\hline Средний возраст“, годы & $41,8 \pm 13,32$ & $40,6-43,0$ & $35,6 \pm 13,4$ & $33,9-37,3$ & 0,0001 \\
\hline $\begin{array}{l}\text { Средний возраст дебюта заболевания*, } \\
\text { годы }\end{array}$ & $34,5 \pm 12,77$ & $33,29-35,61$ & $29,7 \pm 12,89$ & $28,03-31,3$ & 0,0001 \\
\hline $\begin{array}{l}\text { Средняя длительность болезни от } \\
\text { момента установления диагноза*, } \\
\text { годы }\end{array}$ & $7,3 \pm 6,23$ & $6,77-7,90$ & $5,9 \pm 5,08$ & $5,3-6,59$ & 0,0027 \\
\hline Семейный анамнез ВЗК, абс. (\%) & $15(3,2)$ & $1,8-5,3$ & $2(0,8)$ & $0,1-3,0$ & 0,0672 \\
\hline \multicolumn{6}{|l|}{ Занятость, абс. (\%): } \\
\hline работающий & $270(58,1)$ & $53,4-62,6$ & $113(46,9)$ & $40,5-53,4$ & 0,0053 \\
\hline неработающие по разным причинам & $195(41,9)$ & $37,4-46,6$ & $128(53,1)$ & $46,6-59,6$ & \\
\hline
\end{tabular}

ВЗК - воспалительное заболевание кишечника, ДИ - доверительный интервал

"Данные представлены как среднее и стандартное отклонение $(\mathrm{M} \pm \mathrm{SD})$

Доля пациентов, имевших инвалидность по основному заболеванию, также была статистически значимо больше при БК - 56\% против $36,6 \%$ при ЯК (рис. 2).

Диагноз ЯК и БК был верифицирован с помощью стандартных методов, частота применения которых в исследуемом периоде приведена в табл. 3.

\section{Лекарственная терапия}

По результатам ретроспективного анализа глубиной в 2 года до включения пациентов в исследование определены основные схемы лекарственной терапии (табл. 4).

Более четверти пациентов с ЯК получали схемы лечения на основе препаратов из группы 5-АСК в сочетании с глюкокортикостероидами (ГКС) или без них (схема 1). При БК такая схема использовалась статистически значимо реже всего в 3,7\%.

Реже применялись комбинированные схемы с частотой от 13 до 24\% как при ЯК, так и при БК (см. табл. 4):

- 5-АСК с последующим дополнительным назначением иммуносупрессора (ИС) \pm ГКС (схема 5). Частота назначения таких схем при ЯК и БК не различалась;

- стандартная терапия (СТ) включала любой препарат из группы 5-АСК, ИС или ГКС (за исключением генно-инженерного биологического препарата (ГИБП)) с последующим полным
Таблица 2. Характеристика заболевания у исследуемой когорты пациентов с воспалительным заболеванием кишечника на момент включения в исследование INTENT

\begin{tabular}{lccc}
\hline Показатель & $\begin{array}{c}\text { Язвенный колит } \\
(\mathrm{n}=465), \%\end{array}$ & $\begin{array}{c}\text { Болезнь Крона } \\
(\mathrm{n}=241) \%\end{array}$ & $\begin{array}{c}\text { Значение } p \\
\text { (различия }\end{array}$ \\
& $(\mathrm{aбc.)}$ & $(\mathrm{aбc.)}$ & в зависимости \\
& & от нозологии)
\end{tabular}

\begin{tabular}{|c|c|c|c|}
\hline \multicolumn{4}{|l|}{ Протяженность/локализация } \\
\hline тотальный & $58,9(274)$ & & - \\
\hline левосторонний & $33,1(154)$ & & \\
\hline проктит & $8(37)$ & & \\
\hline терминальный илеит & & $23,7(57)$ & \\
\hline илеоколит & & $54,8(132)$ & \\
\hline колит & & $20,3(43)$ & \\
\hline $\begin{array}{c}\text { верхние отделы ЖКТ } \\
\text { (изолированные) }\end{array}$ & & $1,2(3)$ & \\
\hline \multicolumn{4}{|l|}{ Характер течения } \\
\hline рецидивирующий & $72,9(339)$ & $60,6(146)$ & 0,0011 \\
\hline непрерывный & $27,1(126)$ & $39,4(95)$ & \\
\hline $\begin{array}{c}\text { Частота осложнений в анамнезе } \\
\text { в период до } 2 \text { лет к моменту } \\
\text { включения в исследование }\end{array}$ & $12,9(40)$ & $57,4(136)$ & 0,0001 \\
\hline Частота ВКП & $23,4(109)$ & $28,2(68)$ & 0,1705 \\
\hline
\end{tabular}

ВКП - внекишечные проявления, ЖКТ - желудочно-кишечный тракт 


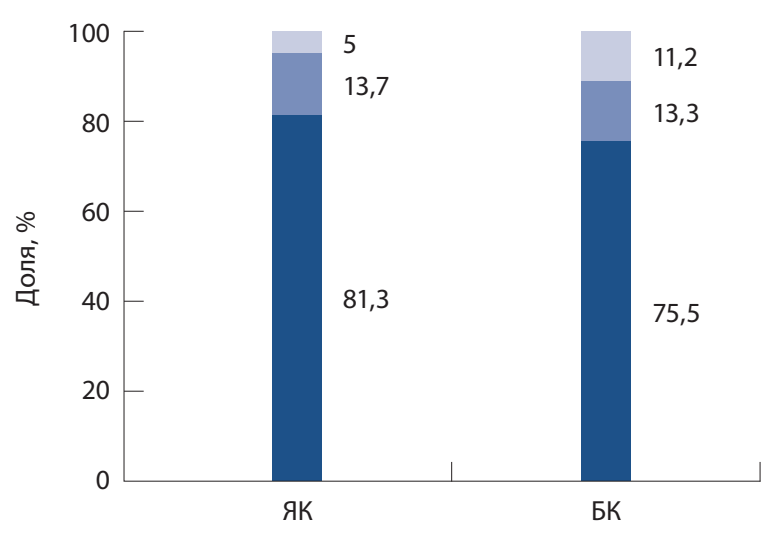

Не курит Курил ранее Курит в настоящее время

Рис. 1. Статус курения у пациентов с воспалительным заболеванием кишечника на момент включения в исследование INTENT; БK - болезнь Крона, ЯК - язвенный колит; $p<0,001$

прекращением лечения либо сокращением до монотерапии ГКС (схема 6). Такой вариант при ЯК применяли статистически значимо чаще, чем при БК (в 14,8\% против 5\%);

- 5-АСК с последующей комбинацией с ИС и затем назначение любого ингибитора фактора некроза опухоли-альфа (иФНО-а) как основного препарата, но при этом больные продолжали принимать аминосалицилаты или ИС либо обе группы препаратов (схема 3). При БК эту схему использовали статистически значимо чаще, чем при ЯК;

- начало лечения с иФНО-а в комбинации с любым препаратом СТ без последующих изменений (схема 8). Эту схему статистически

Таблица 3. Количество пациентов с язвенным колитом и болезнью Крона, которым проводились различные диагностические методы исследования (как минимум единожды в период до 2 лет до включения в исследование INTENT)

\begin{tabular}{lll}
\hline Метод & Язвенный колит, \% & Болезнь Крона, \% \\
\hline Биомаркеры* & 89,9 & 90,9 \\
Эндоскопия & 96,6 & 92,5 \\
Биопсия & 68 & 58,9 \\
Рентген ЖКТ & 20,9 & 36,5 \\
МРТ/КТ & 9,5 & 51,5 \\
УзИ кишечника & 17,2 & 38,2 \\
\hline
\end{tabular}

ЖКТ - желудочно-кишечный тракт, КТ - компьютерная томография, МРТ - магнитно-резонансная томография, УЗИ - ультразвуковое исследование

${ }^{*}$ С-реактивный белок и/или фекальный кальпротектин

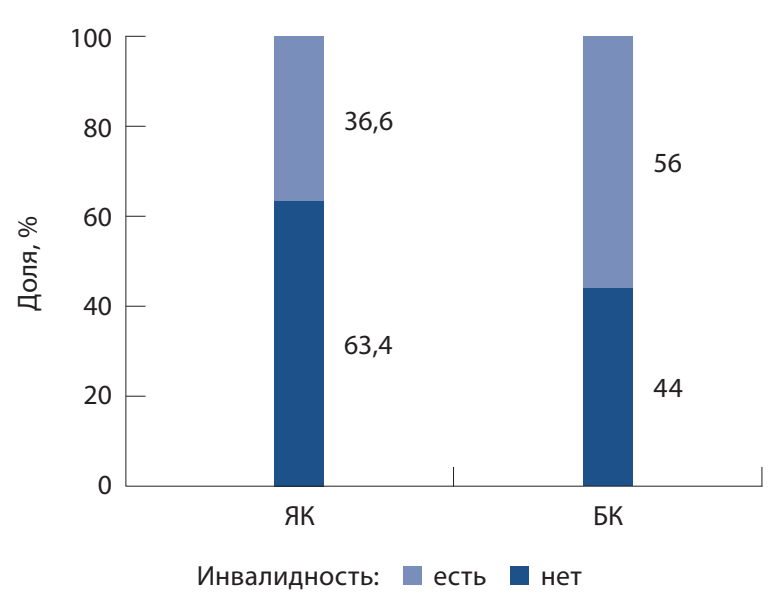

Рис. 2. Статус инвалидности по основному заболеванию у пациентов с воспалительным заболеванием кишечника на момент включения в исследование INTENT; БK - болезнь Крона, ЯК - язвенный колит; $\mathrm{p}<0,05$

значимо чаще применяли при БК (в 24,1\% против 13,6\% при ЯК).

Еще реже применяли следующие терапевтические схемы:

- старт терапии с 5-АСК с последующим назначением ГИБП при сохранении 5-АСК (схема 2). Частота подобного подхода при ЯК и БК не различалась (4,5 и 2,5\% соответственно);

- начало лечения с иФНО-а в сочетании с любым препаратом СТ, затем отмена ГИБП при сохранении СТ или ее отмене (схема 4). Эту схему применяли без значимых статистических различий при ЯК и БК: в 4,3 и 7,5\% случаев соответственно.

Самой частой схемой при ЯК была комбинация 5-АСК и ГКС. Общая частота назначения ГИБП при ЯК (схемы 2, 3, 4, 8, 9) составила 39,4\%. При БК наиболее часто назначались ГИБП в первой линии терапии в сочетании со СТ или без нее $(24,1 \%)$, общая частота применения ГИБП была существенно выше, чем при ЯК и составила 66,4\% (схемы 2, 3, 4, $8,9)$. Ведолизумаб при БК назначали статистически значимо чаще, чем при ЯК (в 10,4 и 3,4\% соответственно, $\mathrm{p}=0,0003)$. Следует отметить, что препараты 5-АСК присутствовали почти во всех схемах лечения БК, что не соответствует отечественным и международным клиническим рекомендациям [2].

Существующие сегодня российские и международные клинические рекомендации предусматривают длительность терапии ГКС не более 12 недель (84 дня), ГКС не используются для поддерживающей терапии. Нежелательны и повторные курсы монотерапии ГКС при рецидиве заболевания $[1,2,11,12]$. 
Таблица 4. Распределение участников исследования INTENT по схемам лекарственной терапии и их длительности

\begin{tabular}{|c|c|c|c|c|}
\hline \multirow[t]{2}{*}{ № $\Pi / \Pi$} & \multirow[t]{2}{*}{ Схема терапии } & \multicolumn{3}{|l|}{ Частота назначения, n (\%) } \\
\hline & & язвенный колит (n=465) & болезнь Крона (n=241) & $\begin{array}{l}\text { значение } p \text { (различия } \\
\text { в зависимости от нозологии) }\end{array}$ \\
\hline 1. & $5-A C K \pm \Gamma K C$ & $118(25,4)$ & $9(3,7)$ & 0,0001 \\
\hline 2. & $5-\mathrm{ACK} \rightarrow$ $\rightarrow \oplus \mathrm{HO}-\mathrm{a} \pm 5-\mathrm{ACK}$ & $21(4,5)$ & $6(2,5)$ & 0,2181 \\
\hline 3. & $\begin{array}{r}5-\mathrm{ACK} \rightarrow \text { } \rightarrow \mathrm{UC}+5-\mathrm{ACK} \rightarrow \\
\text { иФHO- } \pm \pm \text { ИC } \pm 5-\mathrm{ACK}\end{array}$ & $63(13,5)$ & $53(22,0)$ & 0,0052 \\
\hline 4. & иФНО- $а \pm$ СТ $\rightarrow$ отмена ГИБП \pm СТ & $20(4,3)$ & $18(7,5)$ & 0,0812 \\
\hline 5. & $5-A C K \rightarrow$ ИC $+5-A C K \pm Г K C$ & $83(17,9)$ & $55(22,8)$ & 0,1331 \\
\hline 6. & СТ $\rightarrow$ отмена терапии \pm ГКС & $69(14,8)$ & $12(5,0)$ & 0,0001 \\
\hline 7. & ИC $+5-A C K \pm Г K C \rightarrow 5-A C K \pm Г K C$ & $12(2,6)$ & $5(2,0)$ & 0,7994 \\
\hline 8. & иФНО- $\mathrm{a} \pm \mathrm{CT}$ & $63(13,6)$ & $58(24,1)$ & 0,0007 \\
\hline 9. & Схемы, включающие ведолизумаб & $16(3,4)$ & $25(10,4)$ & 0,0003 \\
\hline
\end{tabular}

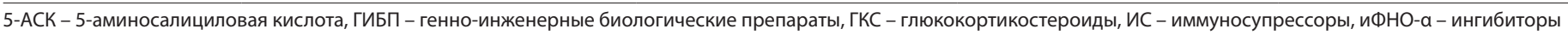
фактора некроза опухоли-а, СТ - стандартная терапия: любой препарат из групп 5-АСК, ИС или ГКС за исключением ГИБП

Таблица 5. Особенности применения глюкокортикостероидов у пациентов с воспалительными заболеваниями кишечника, не получавших биологическую терапию

\begin{tabular}{|c|c|c|}
\hline № & Схема терапии & Длительность применения ГКС, медиана (25-75\%) \\
\hline \multicolumn{3}{|c|}{ Язвенный колит } \\
\hline 1. & $\begin{array}{l}5-A C K \pm \Gamma K C \\
\cdot 5-A C K+\Gamma K C \\
\cdot \Gamma K C\end{array}$ & $\begin{array}{l}\text { Циклы - } 2 \text { (1-7) } \\
\text { Дни - } 117(75,5-206) \\
\text { Дни - } 33 \text { (30-94) }\end{array}$ \\
\hline 2. & $\begin{array}{l}5-A C K \rightarrow \text { } \rightarrow+5-A C K \pm \Gamma K C \\
\cdot 5-A C K+\Gamma K C \\
\cdot И C+5-A C K+\Gamma K C \\
\cdot \Gamma K C\end{array}$ & $\begin{array}{l}\text { Циклы - } 2(1-8) \\
\text { Дни - 186,5 (146,75-217,25) } \\
\text { Дни - } 95(31-146,5) \\
\text { Дни - } 209(165,5-398,0)\end{array}$ \\
\hline 3. & 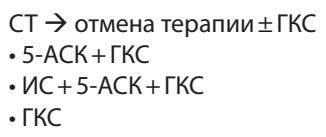 & $\begin{array}{l}\text { Циклы - } 1(1-5) \\
\text { Дни - 104,0 (89,5-131,0) } \\
\text { Дни - } 91(89,5-100,0) \\
\text { Дни - 75,5 (6,5-159,75) }\end{array}$ \\
\hline \multicolumn{3}{|c|}{ Болезнь Крона } \\
\hline 1. & $\begin{array}{l}5-A C K \rightarrow \text { } \rightarrow+5-A C K \pm \Gamma K C \\
\cdot 5-A C K+Г K C \\
\cdot И C+5-A C K+\Gamma K C \\
\cdot \Gamma K C\end{array}$ & $\begin{array}{l}\text { Циклы - } 2(1-5) \\
\text { Дни - } 164(150,5-177,5) \\
\text { Дни - } 93(60-149) \\
\text { Дни - 121,5 (121,25-121,75) }\end{array}$ \\
\hline
\end{tabular}

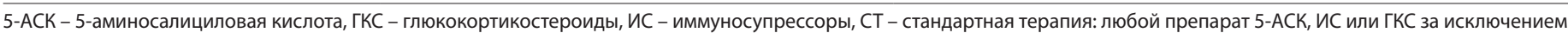
генно-инженерного биологического препарата

Анализ реальной практики применения ГКС позволил выявить ряд особенностей и негативных моментов (табл. 5):

- половина пациентов с ЯК и БК в рамках большинства схем терапии получали более
2 курсов ГКС в течение рассматриваемого периода времени, в ряде случаев от 5 до 8 курсов, то есть почти непрерывно;

- один курс ГКС получали лишь пациенты с ЯК, начавшие лечение со СТ с постепенной ее 
отменой и назначением ГКС или без них (медиана числа курсов равна 1);

- средняя длительность курсов ГКС в большинстве схем как для ЯК, так и для БК колебалась в интервале 91-209 дней, что значительно превышает рекомендуемую продолжительность;

- лишь в единичных схемах продолжительность лечения ГКС соответствовала 33-75 дням.

\section{Обсуждение}

INTENT представляет собой первое крупное исследование клинической практики лечения пациентов с ВЗК средней и тяжелой степени в Российской Федерации, Республике Казахстан и Республике Беларусь. Ретроспективный обзор включал данные по амбулаторному лечению, что также может быть использовано для изучения и критической оценки сложившейся практики. Несмотря на промежуточный характер представленных данных, они могут служить наглядным отражением сложившейся практики лечения пациентов со среднетяжелыми и тяжелыми ВЗК.

Анализ демографических данных показывает, что ВЗК в схожей мере подвержены оба пола. ВЗК дебютируют в большинстве случаев до 40 лет, в нашем исследовании возраст манифестации при БК был в среднем 29,66 года, при ЯК - 34,45 года. Средний период времени от дебюта до включения в исследование при БК составил почти 6 лет по сравнению с 7,3 года при ЯК. Как возраст пациентов на момент включения в исследование, так и возраст манифестации и время от дебюта заболевания до включение в исследование были статистически значимо ниже у больных БК, нежели ЯК. При этом семейный анамнез ВЗК встречался крайне редко. Важным фактором развития и течения ВЗК считается курение. По этому критерию доля больных-курильщиков на момент проведения исследования была статистически значимо выше при БК по сравнению с ЯК, что совпадает с международными данными [13]. В целом демографические показатели пациентов соответствовали мировым тенденциям [14] (см. табл. 1).

Тяжесть течения ВЗК подтверждается изучением характера клинического течения - чуть больше четверти пациентов с ЯК и почти $40 \%$ с БК имели хроническое непрерывное течение (периоды ремиссии более 6 мес. отсутствовали). Частота развития осложнений в период до 2 лет, предшествующих включению в исследование, при ЯК составила $12,9 \%$, тогда как при БК таких пациентов уже более половины. Частота развития внекишечных проявлений при ЯК и БК близка и составляла от четверти до трети всех пациентов.
$60 \%$ пациентов с ЯК имеют обширное поражение кишечника - левосторонний или тотальный колит. 55\% пациентов с БК имели поражение подвздошной и толстой кишки (илеоколит). Частота осложнений и характер течения ВЗК рассмотренной когорты в целом согласуются с данными международных эпидемиологических исследований [15].

Анализ диагностической практики показывает, что изучение биомаркеров (С-реактивного белка, фекального кальпротектина) как минимум единожды в течение двух лет до момента включения в исследование применялось у 89,9\% при ЯК и 90,9\% при БК.

Изучение практики лекарственной терапии позволило получить ценные результаты. Большинство пациентов с ЯК получают стандартную терапию, в то время как пациенты с БК чаще биологическую терапию ГИБП. Основная часть ГИБП приходилась на препараты из групп иФНО-а, которые суммарно получали более $40 \%$ участников рассмотренной когорты пациентов с ЯК и БК. У 74,9\% пациентов (76,6\% больных ЯК и 71,8\% больных БК) не было зарегистрировано внекишечных проявлений, но, несмотря на это, на кишечно-селективную биологическую терапию - препарат ведолизумаб - приходилось около $6 \%$ (3,4\% при ЯК и 10,4\% при БК) участников. Одним из критериев целесообразности назначения ГИБП может быть потребность в 2 и более курсах ГКС $[16,17]$, а как видно из представленных результатов, на практике часто назначение курсов стероидной терапии фиксировали по 2 и более раза в анамнезе у пациентов, которым никогда не назначалась биологическая терапия, что указывает на значительную неудовлетворенную потребность в ГИБП.

В практике лекарственной терапии БК, несмотря на высокую частоту использования ГИБП, по-прежнему распространено применение для поддержания ремиссии препаратов 5-АСК, которые признаны недостаточно эффективными при лечении среднетяжелых и тяжелых форм БК [18-20] и в Клинических рекомендациях 2020 г. исключены из перечня средств терапии, рекомендуемых для поддержания ремиссии [2].

Самым критичным аспектом сложившейся практики следует признать назначение ГКС, избыточное и по частоте, и по длительности применения, в том числе у пациентов, которым никогда не назначалась биологическая терапия. При рекомендованном ограничении приема ГКС в 12 недель в большинстве случаев данная рекомендация не соблюдается, значительная доля участников принимала ГКС более полугода. 
Полученные результаты предоставляют богатый материал для анализа. Высокая частота осложнений, значимое число пациентов с прогрессирующим течением и обширным поражением ЖКТ, а также распространенность неоптимальных схем терапии служат отражением недостатков сложившейся практики, которые необходимо исправить. Выявленные особенности медикаментозного лечения в реальной клинической практике могут быть использованы для коррекции клинических рекомендаций в будущем. Полученные данные также ясно указывают на наличие потребности в повышении информированности врачей в отношении современных подходов к терапии ВЗК.

\section{Заключение}

Исследование INTENT впервые позволило достаточно подробно изучить текущую клиническую практику лечения пациентов со среднетяжелыми и тяжелыми ВЗК в Российской Федерации, Республике Беларусь и Республике Казахстан. Несмотря на промежуточный характер представленных данных, они уже позволяют сделать ряд важных выводов для совершенствования медицинской помощи при ВЗК:

\section{Дополнительная информация}

\section{Финансирование и конфликт интересов}

Исследование и представленный анализ были проведены при организационной и финансовой поддержке ООО «Такеда Фармасьютикалс».

\section{Участие авторов}

О.В. Князев и Е.А. Белоусова - разработка дизайна клинической части исследования, анализ и интерпретация результатов, концепция и дизайн статьи, редактирование текста, утверждение итогового варианта текста рукописи; Д.И. Абдулганиева, И.В. Губонина, Д.А. Кайбуллаева,
- относительно часто встречаются ошибочные стратегии лечения пациентов с ВЗК (например, отмена терапии после достижения ремиссии - см. паттерны 4 и 6 в табл. 4), что может быть вызвано как недостаточной информированностью врачей о современных подходах (в том числе персонифицированном подходе) к терапии, так и недостаточной приверженностью пациентов к назначенной терапии;

- в связи с недостаточной решительностью лечащих врачей в принятии решения о назначении биотерапии необходимо дальнейшее разъяснение рисков чрезмерного необоснованного использования стероидов и позднего назначения ГИБП, распространение данных о преимуществах биологической терапии при своевременном ее назначении;

- учитывая, что у большинства пациентов не обнаружено внекишечных проявлений, а данные многоцентровых исследований $[21,22]$ показали преимущество назначения ведолизумаба у бионаивных пациентов, при этом не оказывающего влияния на эффективность последующих линий биотерапии [23, 24], представляется логичным активное внедрение в клиническую практику кишечно-селективной терапии. (;)

\section{Литература / References}

1.Ивашкин ВТ, Шелыгин ЮА, Белоусова ЕА, Абдулганиева ДИ, Алексеева ОА, Ачкасов СИ, Валуйских ЕЮ, Варданян АВ, Веселов АВ, Веселов ВВ, Головенко ОВ, Губонина ИВ, Жигалова ТН, Кашников ВН, Князев ОВ, Макарчук ПА, Москалев АИ, Нанаева БА, Низов АА, Никитина НВ, Николаева НН, Павленко ВВ, Полуэктова ЕА, Светлова ИО, Тарасова ЛВ, Ткачев АВ, Фролов СА, Хлынова ОВ, Чашкова ЕЮ, Шапина MB, Шептулин AA, Шифрин ОС, Щукина ОБ. Проект клинических рекомендаций по диагностике и лечению язвенного колита. Колопроктология. 2019;18(4):7-36. doi: 10.33878/2073-7556-2019-18-4-7-36. [lvash- kin VT, Shelygin YuA, Belousova EA, Abdulganieva DI, Alekseeva OA, Achkasov SI, Valuiskikh EYu, Vardanyan AV, Veselov AV, Veselov VV, Golovenko OV, Gubonina IV, Zhigalova TN, Kashnikov VN, Knyazev OV, Makarchuk PA, Moskaliev Al, Nanaeva BA, Nizov AA, Nikitina NV, Nikolaeva NN, Pavlenko VV, Poluektova EA, Svetlova IO, Tarasova LV, Tkachev AV, Frolov SA, Khlynova OV, Chashkova EYu, Shapina MV, Sheptulin AA, Shifrin OS, Shchukina OB. [Project: clinical guidelines for the diagnostics and treatment of ulcerative colitis]. Koloproktologia [Coloproctology]. 2019;18(4): 7-36. Russian. doi: 10.33878/2073-7556-201918-4-7-36.]
2. Клинические рекомендацииподиагностике и лечению болезни Крона у взрослых (проект). Колопроктология. 2020;19(2):8-38. doi: $\quad 10.33878 / 2073-7556-2020-19-2-8-38$. [Crohn's disease. Clinical recommendations (preliminary version). Koloproktologia [Coloproctology]. 2020;19(2):8-38. Russian. doi: 10.33878/2073-7556-2020-19-2-8-38.]

3. Князев ОВ, Шкурко ТВ, Фадеева НА, Бакулин ИГ, Бордин ДС. Эпидемиология хронических воспалительных заболеваний кишечника. Вчера, сегодня, завтра. Экспериментальная и клиническая гастроэнтерология. 2017;(3):4-12. [Knyazev OV, Shkurko TV, Fadeyeva NA, Bakulin IG, Bor- 
din DS. [Epidemiology of chronic inflammatory bowel disease. Yesterday, today, tomorrow]. Experimental and Clinical Gastroenterology. 2017;(3):4-12. Russian.]

4.Плотникова ЕЮ, Чашкова ЕЮ. Некоторые аспекты лечения воспалительных заболеваний кишечника. Лечащий врач. 2019;(8): 14-20. [Plotnikova EYu, Chashkova EYu. [Certain aspects for treatment of inflammatory bowel disease]. Lechashchiy Vrach [Attending Physician]. 2019;(8):14-20. Russian.]

5.Горгун ЮВ, Боровнёва ЕА, Столярова ТА, Юркевич АС, Жарская ОМ, Владимирова ИА, Саевич НИ. Хронические воспалительные заболевания кишечника в Республике Беларусь. Лечебное дело. 2017;3(55): 5-12. [Gorgun JV, Barauniova KA, Stolyarova TA, Yurkevich AS, Zharskaya OM, Saevich NI, Vladimirova IA. [Chronic inflammatory bowel disease in the Republic of Belarus]. Lechebnoe Delo [Medical Care]. 2017;3(55):5-12. Russian.]

6. Kaibullayeva J, Ualiyeva A, Oshibayeva A, Dushpanova A, Marshall JK. Prevalence and patient awareness of inflammatory bowel disease in Kazakhstan: a cross-sectional study. Intest Res. 2020;18(4):430-437. doi: 10.5217/ ir.2019.00099.

7.Белоусова ЕА, Абдулганиева дИ, Алексеева ОП, Алексеенко СА, Барановский АЮ, Валуйских ЕЮ, Головенко АО, Головенко ОВ, Жигалова ТН, Князев ОВ, Куляпин АB, Лахин АB, Ливзан МА, Лубянская ТГ, Николаева НН, Никитина НВ, Никулина ИВ, Осипенко МФ, Павленко ВВ, Парфенов АИ, Рогачиков ЮЕ, Светлова ИО, Ткачев АВ, Ткаченко ЕИ, Халиф ИЛ, Чашкова ЕЮ, Щукина ОБ, Язенок НС, Яковлев АА. Социально-демографическая характеристика, особенности течения и варианты лечения воспалительных заболеваний кишечника в России. Результаты двух многоцентровых исследований. Альманах клинической медицины. 2018;46(5): 445-463. doi: 10.18786/2072-0505-2018-465-445-463. [Belousova EA, Abdulganieva DI, Alexeeva OP, Alexeenko SA, Baranovsky AYu, Valuyskikh EYu, Golovenko AO, Golovenko OV, Zhigalova TN, Knyazev OV, Kulyapin $\mathrm{AV}$, Lakhin $\mathrm{AV}$, Livzan MA, Lubyanskaya TG, Nikolaeva NN, Nikitina NV, Nikulina IV, Osipenko MF, Pavlenko VV, Parfenov Al, Rogachikov YE, Svetlova IO, Tkachev AV, Tkachenko El, Khalif IL, Chashkova EYu, Shchukina OB, Yazenok NS, Yakovlev AA. [Social and demographic characteristics, features of disease course and treatment options of inflammatory bowel disease in Russia: results of two multicenter studies]. Almanac of Clinical Medicine. 2018;46(5):
445-463. Russian. doi: 10.18786/2072-05052018-46-5-445-463.]

8. Халиф ИЛ, Шапина МВ, Головенко АО, Белоусова ЕА, Чашкова ЕЮ, Лахин АВ, Князев ОВ, Барановский АЮ, Николаева НН, Ткачев АВ. Течение хронических воспалительных заболеваний кишечника и методы их лечения, применяемые в Российской Федерации (Результаты многоцентрового популяционного одномоментного наблюдательного исследования). Российский журнал гастроэнтерологии, гепатологии, колопроктологии. 2018;28(3):54-62. doi: 10.22416/1382-4376-2018-28-3-54-62. [Khalif IL, Shapina MV, Golovenko AO, Belousova YA, Chashkova YYu, Lakhin AV, Knyazev OV, Baranovsky AYu, Nikolayeva NN, Tkachev AV. [Chronic inflammatory bowel diseases: the course and treatment methods in Russian Federation (Results of multicenter population-based one-stage observational study)]. Russian Journal of Gastroenterology, Hepatology, Coloproctology. 2018;28(3):54-62. Russian. doi: 10.22416/1382-4376-2018-28-354-62.]

9. Belousova E, Khalif I. Tu1290 Social, Demographic and Clinical Features of Inflammatory Bowel Disease in Russia. Gastroenterology. 2012;142(5 Suppl 1):S-794. doi: 10.1016/ S0016-5085(12)63083-2.

10. World Medical Association. World Medical Association Declaration of Helsinki: ethical principles for medical research involving human subjects. JAMA. 2013;310(20):2191-2194. doi: 10.1001/jama.2013.281053.

11. Harbord $M$, Eliakim $R$, Bettenworth $D$, Karmiris K, Katsanos K, Kopylov U, Kucharzik T, Molnár T, Raine T, Sebastian S, Tavares de Sousa H, Dignass A, Carbonnel F, European Crohn's and Colitis Organisation [ECCO]. Third European Evidence-based Consensus on Diagnosis and Management of Ulcerative Colitis. Part 2: Current Management. J Crohns Colitis. 2017;11(7):769-784. doi: 10.1093/ecco-jcc/ jjx009.

12. Torres J, Bonovas S, Doherty G, Kucharzik T, Gisbert JP, Raine T, Adamina M, Armuzzi A, Bachmann O, Bager P, Biancone L, Bokemeyer B, Bossuyt P, Burisch J, Collins P, El-Hussuna A, Ellul P, Frei-Lanter C, Furfaro F, Gingert C, Gionchetti P, Gomollon F, González-Lorenzo M, Gordon H, Hlavaty T, Juillerat P, Katsanos K, Kopylov U, Krustins E, Lytras T, Maaser C, Magro F, Marshall JK, Myrelid P, Pellino G, Rosa I, Sabino J, Savarino E, Spinelli A, Stassen L, Uzzan M, Vavricka S, Verstockt B, Warusavitarne J, Zmora O, Fiorino G. ECCO Guidelines on Therapeutics in Crohn's Disease: Medical Treatment. J Crohns Colitis. 2020;14(1):4-22. doi: 10.1093/ ecco-jcc/jjz180.
13. Parkes GC, Whelan K, Lindsay JO. Smoking in inflammatory bowel disease: Impact on disease course and insights into the aetiology of its effect. J Crohn's Colitis. 2014;8(8):717-725. doi: 10.1016/j.crohns.2014.02.002.

14. Mak WY, Zhao M, Ng SC, Burisch J. The epidemiology of inflammatory bowel disease: East meets west. J Gastroenterol Hepatol. 2020;35(3):380-389. doi: 10.1111/jgh.14872.

15. Levine JS, Burakoff R. Extraintestinal manifestations of inflammatory bowel disease. Gastroenterol Hepatol (N Y). 2011;7(4):235-241.

16. Siegel $C A$, Whitman $C B$, Spiegel BMR, Feagan $B$, Sands $B$, Loftus Jr EV, Panaccione $R$, D'Haens G, Bernstein CN, Gearry R, Ng SC, Mantzaris GJ, Sartor B, Silverberg MS, Riddell $R$, Koutroubakis IE, O'Morain C, Lakatos PL, McGovern DPB, Halfvarson J, Reinisch W, Rogler G, Kruis W, Tysk C, Schreiber S, Danese S, Sandborn W, Griffiths A, Moum B, Gasche C, Pallone F, Travis S, Panes J, Colombel JF, Hanauer S, Peyrin-Biroulet L. Development of an index to define overall disease severity in IBD. Gut. 2018;67(2):244-254. doi: 10.1136/gutjnl-2016-312648.

17. Peyrin-Biroulet L, Panés J, Sandborn WJ, Vermeire S, Danese S, Feagan BG, Colombel JF, Hanauer SB, Rycroft B. Defining Disease Severity in Inflammatory Bowel Diseases: Current and Future Directions. Clin Gastroenterol Hepatol. 2016;14(3):348-354.e17. doi: 10.1016/j.cgh.2015.06.001.

18. Gomollón F, Dignass A, Annese V, Tilg H, Van Assche G, O Lindsay J, Peyrin-Biroulet L, Cullen GJ, Daperno M, Kucharzik T, Rieder F, Almer S, Armuzzi A, Harbord M, Langhorst J, Sans M, Chowers $Y$, Fiorino $G$, Juillerat $P$, Mantzaris GJ, Rizzello F, Vavricka S, Gionchetti P, ECCO. $3^{\text {rd }}$ European Evidence-based Consensus on the Diagnosis and Management of Crohn's Disease 2016: Part 1: Diagnosis and Medical Management. J Crohns Colitis. 2017;11(1):3-25. doi: 10.1093/ecco-jcc/ jjw168.

19. Matsuoka K, Kobayashi T, Ueno F, Matsui T, Hirai F, Inoue N, Kato J, Kobayashi K, Kobayashi K, Koganei K, Kunisaki R, Motoya S, Nagahori M, Nakase H, Omata F, Saruta M, Watanabe T, Tanaka T, Kanai T, Noguchi $Y$, Takahashi KI, Watanabe K, Hibi T, Suzuki Y, Watanabe M, Sugano K, Shimosegawa T. Evidence-based clinical practice guidelines for inflammatory bowel disease. J Gastroenterol. 2018;53(3):305-353. doi: 10.1007/s00535018-1439-1.

20. Lamb CA, Kennedy NA, Raine T, Hendy PA, Smith PJ, Limdi JK, Hayee BH, Lomer MCE, Parkes GC, Selinger C, Barrett KJ, Davies RJ, 
Bennett C, Gittens S, Dunlop MG, Faiz O, Fraser A, Garrick V, Johnston PD, Parkes M, Sanderson J, Terry $\mathrm{H}$, IBD guidelines eDelphi consensus group; Gaya DR, lqbal TH, TayIor SA, Smith M, Brookes M, Hansen R, Hawthorne AB. British Society of Gastroenterology consensus guidelines on the management of inflammatory bowel disease in adults. Gut.
2019;68 Suppl 3:s1-s106. doi: 10.1136/gutjnl-2019-318484.

21. Feagan BG, Rubin DT, Danese $S$, Vermeire $S$, Abhyankar B, Sankoh S, James A, Smyth M. Efficacy of Vedolizumab Induction and Maintenance Therapy in Patients With Ulcerative Colitis, Regardless of Prior Exposure to Tumor Necrosis Factor Antagonists. Clin Gastroen- terol Hepatol. 2017;15(2):229-239.e5. doi: 10.1016/j.cgh.2016.08.044.

22. Sands BE, Sandborn WJ, Van Assche G, Lukas M, Xu J, James A, Abhyankar B, Lasch K. Vedolizumab as Induction and Maintenance Therapy for Crohn's Disease in Patients Naïve to or Who Have Failed Tumor Necrosis Factor Antagonist. Therapy Inflamm

\title{
Real world practice of medical treatment for moderate and severe inflammatory bowel diseases in Russian Federation, Republic of Belarus and Republic of Kazakhstan: intermediate results of the INTENT study
}

\author{
O.V. Knyazev' • E.A. Belousova² • D.I. Abdulganieva3 •I.V. Gubonina ${ }^{4}$ J.A. Kaibullayeva ${ }^{5}$ \\ Yu.Kh. Marakhouski $\bullet$ E.Yu. Chashkova $•$ M.V. Shapina ${ }^{8} \cdot$ O.B. Shchukina ${ }^{9} \cdot$ B.B. Gegenava ${ }^{10} \cdot$ \\ N.S. Oliferuk ${ }^{10}$
}

\begin{abstract}
Background: The analysis of data obtained from real world clinical practice of management of patients with inflammatory bowel diseases (IBD) is an effective tool to improve medical care for this patient category. Studies of the kind are rare in Russia, which hinders a critical assessment of the current situation and optimization of the established approaches.

Aim: To study real world practice of medical treatment of patients with moderate and severe IBD in the Russian Federation, Republic of Belarus and Republic of Kazakhstan.
\end{abstract}

Materials and methods: We analyzed intermediate results from the INTENT study (NCT03532932), which is a multinational, multicenter, retrospective and prospective, non-interventional observation trial being performed in the Russian Federation, Republic of Belarus and Republic of Kazakhstan. The retrospective analysis included data from 706 patients above 18 years of age with confirmed diagnosis of moderate/severe ulcerative colitis (UC) and Crohn's disease (CD) made at least 2 years before the study entry, with acute exacerbations of the disease at the study entry or within the last 2 years. Data were collected during routine management; at the study entry the patients were treated with a standard regimen including 5-aminosalicylic acid (5-ASA) agents, glucocorticosteroids (GCS) and immunosuppressants (IS), as well as genetically engineered biological agents (GEBA).

Results: Among 706 IBD patients, 465 had UC and 241 had CD. The male to female ratios in both groups were similar. Mean age of the UC patients was higher than that of the $C D$ patients (41.8 [95\% confidence interval $(\mathrm{Cl}) 40.6-43.0$ ] years vs $35.6[95 \% \mathrm{Cl} 33.9-37.3]$ years, $p=0.0001)$. The same difference was noted for the mean age of disease manifestation $(34.5$ [95\% Cl 33.29-35.61] vs 29.7 [ $95 \% \mathrm{Cl} 28.03-31.3$ ] years, $p=0.0001$ ) and for mean duration of disease from the time of diagnosis to the study entry $(7.3$ [95\% Cl 6.77-7.9] for UC and 5.9 [95\% Cl 5.3-6.59] years for $C D$ $p=0.0027)$. The proportion of patients with familial history of IBD was low (3.2 and $0.8 \%$, respectively, $p=0.0672$ ). The number of smokers with $C D$ was more than 2 -fold higher than those with UC $(11.2 \%$ vs $5 \%, \mathrm{p}<0.001) .58 .1 \%$ of the patients in the UC group and $47.0 \%$ of those from the CD group were employed $(p<0.05) .36 .6 \%$ of the UC patients and $56.0 \%$ of the $C D$ patients had the legal disability status due to underlying disease $(p<0.005)$.

The relapsing course of the disease was noted in $72.9 \%$ with UC and $60.6 \%$ with CD, while in the rest of the patients the disease had the continuous course. The degree of UC involvement corresponded to pancolitis in $58.9 \%$ of the cases, to left-sided colitis in $33.1 \%$, and to proctitis in $8 \%$. The distribution of CD location was as follows: ileocolitis $54.8 \%$, terminal ileitis $23.7 \%$, colitis $20.3 \%$, isolated upper gastrointestinal tract involvement $1.2 \%$. The prevalence of complicated UC was $12.9 \%$, and that of the complicated CD $57.4 \%(p=0.0001)$. There were no difference in the rate of extraintestinal manifestations between UC and CD (23.4 and $28.2 \%$, respectively, $\mathrm{p}=0.1705$ ).

$\mathrm{UC}$ and $\mathrm{CD}$ groups differed by their treatment patterns. In the UC group, 5-ASA+GCS regimens were given to $25.4 \%$ of the patients, whereas in the CD group, to $3.7 \%(p \leq 0.0001)$. The second frequent regimens were: 5 -ASA with subsequent IS \pm GCS (17.9\% in UC, $22.8 \%$ in CD, $p=0.1331)$; standard regimen (any 5-ASA agent, IS or GCS, but not GEBA) with subsequent treatment withdrawal or its reduction to GCS monotherapy (14.8\% in UC, $5 \%$ in $C D, p=0.0001) ; 5$-ASA with a subsequent combination with IS, then any tumor necrosis factor- $a$ inhibitor (iTNF- $a$ ) as the basic treatment with continuation of 5-ASA and/or IS (22\% in CD vs $13.5 \%$ in UC, $p=0.0052$ ); treatment initiation from iTNF-a combined with any standard agent without any subsequent modification $(24.1 \%$ in $C D$ and $13.6 \%$ in UC, $p=0.0007$ ). Less frequent the following treatment regimens were used: initial treatment with 5-ASA and subsequent iTNF- $a$ with continuation of 5-ASA ( $4.5 \%$ in UC and $2.5 \%$ in CD, $p=0.2181$ ); initial treatment with iTNF- $a$ in combination with any standard agent with subsequent GEBA withdrawal and continuation of a standard regimen or its withdrawal $4.3 \%$ in UC and $7.5 \%$ in $C D, p=0.0812$ ).

The cumulative frequency of GEBA administration at various stages of treatment for CD (66.4\%) was significantly higher than for UC (39.4\%). Vedolizumab for CD was administered more frequently than for UC (10.4 and $3.4 \%$, respectively, $p=0.0003$ ). The analysis of habitual GCS use revealed a number of negative trends, namely, half of the IBD patients received more than 2 GCS courses within 2 years, and in some cases the number of GCS courses amounted to 5-8. Mean duration of a GCS course in most regimens for UC and CD was in the range of 91 to 209 days, which is significantly higher than the recommended treatment duration of 12 weeks ( 83 days).

Conclusion: With a number of its demographic characteristics and clinical particulars, the study cohort of patients with IBD is compatible to global trends: the male to female ratio, mean patients' age, young age at disease manifestation, smoking status, prevalence of extraintestinal manifestations and the location of CD. It is of note that 5-ASA is included into almost all treatment regimens for $C D$, which does not meet the treatment strategy 
Bowel Dis. 2017;23(1):97-106. doi: 10.1097/ MIB.0000000000000979.

23. Bressler B, Yarur A, Kopylov U, Bassel M, Brett N, Lissoos T, Lopez C, Natsios A, Saha S, Kifnidi C, Demuth D, Patel H, Mantzaris GJ. Abstract P1091. Clinical Effectiveness of First-Line Anti-TNF Therapies and Second-Line Anti-TNF Therapy Post-Vedolizum-

recommended in the guidelines. Frequent administration and long duration of GCS therapy also is in contradiction with the guidelines. Of significant concern is rather rare and late administration of GEBA, especially for UC. We believe that the identified pitfalls are associated both with low awareness of doctors on the current strategies of IBD management and with low patients' compliance to treatment.

Key words: inflammatory bowel diseases, Crohn's disease, ulcerative colitis, epidemiology, medical treatment, real world clinical practice, 5-aminosalicylic acid, glucocorticosteroids, immunosuppressants, genetically engineered biological agents, vedolizumab

For citation: Knyazev OV, Belousova EA, Abdulganieva DI, Gubonina IV, Kaibullayeva JA, Marakhouski YuKh, Chashkova EYu, Shapina MV, Shchukina OB, Gegenava BB, Oliferuk NS. Real world practice of medical treatment for moderate and severe inflammatory bowel diseases in Russian Federation, Republic of Belarus and Republic of Kazakhstan: intermediate results of the INTENT study. Almanac of Clinical Medicine. 2021;49(7):443-454. doi: 10.18786/2072-0505-2021-49-061.

Received 29 September 2021; revised 24 October 2021; accepted 30 October 2021; published online 16 December 2021

\section{Funding and conflict of interests}

The study and its analysis were performed under administrative and financial support from Takeda Pharmaceuticals Ltd.

\section{Authors' contributions}

O.V. Knyazev and E.A. Belousova, design of the clinical part of the study, analysis and interpretation of the results, the paper concept and design, text editing, approval of the final version of the manuscript; D.I. Abdulganieva, I.V. Gubonina, J.A. Kaibullayeva, Yu.Kh. Marakhouski, E.Yu. Chashkova, and M.V. Shapina, design of the clinical part of the study, analysis and interpretation of the results, text writing and editing; O.B. Shchukina, B.B. Gegenava, and N.S. Oliferuk, the study concept and design, data collection and management, data analysis, text writing. All the authors have read and approved the final version of the manuscript before submission, agreed to be accountable for all aspects of the work in ensuring that questions related to the accuracy or integrity of any part of the work are appropriately investigated and resolved. ab Discontinuation in Patients with Ulcerative Colitis or Crohn's Disease. In: United European Gastroenterology. Therapeutic Drug Monitoring. Abstracts 2019. Barcelona, Oct 19-23, 2019.

24. Bar-Gil Shitrit A, Ben-Ya'acov A, Siterman M, Waterman M, Hirsh A, Schwartz D, Zittan E, Adler Y, Koslowsky B, Avni-Biron I, Chow-

Oleg V. Knyazev - MD, PhD, Head of Department of Inflammatory Bowel Diseases'; ORCID: https://orcid. org/0000-0001-7250-0977

$\triangle 86$ Entuziastov shosse, Moscow, 111123, Russian Federation. Tel.: +7 (909) 6911016 .

E-mail: oleg@bk.ru

Elena A. Belousova - MD, PhD, Professor, Head of Department of Gastroenterology; Head of Chair of Gastroenterology, Postgraduate Training Faculty2; ORCID: https://orcid.org/0000-0003-4523-3337. E-mail: eabelous@yandex.ru

Diana I. Abdulganieva - MD, PhD, Professor, First Vice-rector, Head of Chair of Hospital Therapy3; ORCID: https://orcid.org/0000-0001-7069-2725.

E-mail: diana_s@mail.ru

Irina V. Gubonina - MD, PhD, Associate Professor, $2^{\text {nd }}$ Therapy Chair of Postgraduate Education ${ }^{4}$; ORCID: https://orcid.org/0000-0002-6302-7767. E-mail: giv70@bk.ru

Jamilya A. Kaibullayeva - MD, PhD, Associate Professor, Chair of Gastroenterology $y^{5}$ ORCID: https:// orcid.org/0000-0002-0783-4441.

E-mail: kaibullaev@mail.ru ers $\mathrm{Y}$, Ron $\mathrm{Y}$, Israeli $\mathrm{E}$, Ungar B, Yanai $\mathrm{H}$, Maharshak N, Ben-Horin S, Eliakim R, Dotan I, Goldin E, Kopylov U. Safety and effectiveness of ustekinumab for induction of remission in patients with Crohn's disease: A multicenter Israeli study. United European Gastroenterol J. 2020;8(4):418-424. doi: $10.1177 / 2050640620902956$.

Yury Kh. Marakhouski - MD, PhD, Professor, Head of Chair of Gastroenterology and Nutritiology ${ }^{6}$; ORCID: https://orcid.org/0000-0001-7327-7762. E-mail: marakhouski@yahoo.co.uk

Elena Yu. Chashkova - MD, PhD, Coloproctologist, Leading Research Fellow, Head of Laboratory of Reconstructive Surgery, Scientific Department of Clinical Surgery ${ }^{7}$; ORCID: https://orcid.org/00000002-7953-6523. E-mail: elenachash1027@yandex.ru

Marina V. Shapina - MD, PhD, Head of Department of Research in Inflammatory and Functional Bowel Disorders ${ }^{8}$; ORCID: https://orcid.org/0000-0003-11726221. E-mail: shapina.mv@yandex.ru

Oksana B. Shchukina - MD, PhD, Head of Center of Inflammatory Bowel Disease ${ }^{9}$. E-mail: burmao@gmail.com

Badri B. Gegenava - MD, PhD, Medical Advisor ${ }^{10}$; ORCID: https://orcid.org/0000-0002-6701-9780. E-mail: badri.gegenava@takeda.com

Natalya S. Oliferuk - MD, PhD, Therapeutic Area Head (Gastroenterology) ${ }^{10}$.

E-mail: natalya.oliferuk@takeda.com
${ }^{1}$ The Loginov Moscow Clinical Scientific Center; 86-6 Entuziastov shosse, Moscow, 111123, Russian Federation ${ }^{2}$ Moscow Regional Research and Clinical Institute (MONIKI); 61/2 Shchepkina ul., Moscow, 129110, Russian Federation

${ }^{3}$ Kazan State Medical University; 49 Butlerova ul., Kazan, 420012, Russian Federation

${ }^{4}$ Military Medical Academy named after S.M. Kirov; 6A Akademika Lebedeva ul., Saint Petersburg, 194044, Russian Federation

${ }^{5}$ Research Institute of Cardiology and Internal Medicine; 120/25 Ayteke bi, Almaty, 050000, Republic of Kazakhstan ${ }^{6}$ Belarusian Medical Academy of Postgraduate Education; 3-3 P. Brovki ul., Minsk, 220013, Republic of Belarus ${ }^{7}$ Irkutsk Scientific Centre of Surgery and Traumatology; 1 Bortsov Revolyutsii ul., Irkutsk, 664003, Russian Federation ${ }^{8}$ National Medical Research Centre for Coloproctology named after A.N. Ryzhikh; 2 Salyama Adilya ul., Moscow, 123423, Russian Federation

${ }^{9}$ Academician I.P. Pavlov First St. Petersburg State Medical University; 6-8 L'va Tolstogo ul., Saint Petersburg, 197022, Russian Federation

${ }^{10}$ Takeda Pharmaceutical Company Limited; 2-1 Usacheva ul., Moscow, 119048, Russian Federation 